\title{
Broadly tunable high-power random fibre laser
}

\author{
Sergey A. Babin ${ }^{\mathrm{a}}$, Atalla E. El-Taher ${ }^{\mathrm{b}}$, Paul Harper ${ }^{\mathrm{b}}$, Evgeniy V. Podivilov ${ }^{\mathrm{a}}$, Sergei K. Turitsyn ${ }^{\mathrm{b}}$ \\ ${ }^{a}$ Institute of Automation and Electrometry, 1, Ac. Koptuyg ave., Novosibirsk, 630090, Russia \\ ${ }^{\mathrm{b}}$ Aston university, Aston triangle, Birmingham B4 7EZ, UK
}

\begin{abstract}
As shown recently, a long telecommunication fibre may be treated as a natural one-dimensional random system, where lasing is possible due to a combination of random distributed feedback via Rayleigh scattering by natural refractive index inhomogeneities and distributed amplification through the Raman effect. Here we present a new type of a random fibre laser with a narrow $(\sim 1 \mathrm{~nm})$ spectrum tunable over a broad wavelength range $(1535-1570 \mathrm{~nm})$ with a uniquely flat $(\sim 0.1 \mathrm{~dB})$ and high $(>2 \mathrm{~W})$ output power and prominent $(>40 \%)$ differential efficiency, which outperforms traditional fibre lasers of the same category, e.g. a conventional Raman laser with a linear cavity formed in the same fibre by adding point reflectors. Analytical model is proposed that explains quantitatively the higher efficiency and the flatter tuning curve of the random fiber laser compared to conventional one. The other important features of the random fibre laser like "modeless" spectrum of specific shape and corresponding intensity fluctuations as well as the techniques of controlling its output characteristics are discussed. Outstanding characteristics defined by new underlying physics and the simplicity of the scheme implemented in standard telecom fibre make the demonstrated tunable random fibre laser a very attractive light source both for fundamental science and practical applications such as optical communication, sensing and secure transmission.
\end{abstract}

Keywords: Keywords: Fiber laser, Rayleigh scattering, Raman gain, random, distributed feedback, tunable, narrow-line

\section{INTRODUCTION}

Physics of light interaction with amplifying disordered optical media attracts a great deal of interest as a fascinating interdisciplinary field focusing on the development of laser sources based on new principles. Random lasers operating without traditional cavity are demonstrated in a number of configurations with various materials (for a review see [1-7]), on the base of early ideas [8,9]. The operation principles of random lasers are quite different to that ones in a standard laser scheme utilizing an optical cavity to create a positive feedback. The cavity is usually formed by highly-reflecting mirrors which provide multiple round trips of the light through the gain media. Lasing occurs when the integral gain overcomes the cavity loss at the round trip. Typically, operational characteristics of conventional lasers depend on the resonator design that defines the structure of laser modes. On the contrary, in random lasers without well-defined cavity the multiple scattering of photons in an amplifying disordered medium increases the effective optical path, resulting eventually in lasing. The output characteristics of random lasers are shown to be determined by the randomly embedded local spatial modes that may coexist with non-localized extended modes $[6,7]$.

Random lasers have clear advantages including simple technology without the need to engineer a precise cavity, e.g. just a semiconductor powder may be used in diode lasers, see [5]. However, for many applications their current performance, that typically features pulsed operation with complex non-localized emission spectra and accidental direction of the output beam, has to be significantly improved to practically challenge conventional lasers. Various schemes are proposed and applied to improve the performance of random lasers with the goal of achieving stationary operation with beam quality comparable to those in conventional lasers. Promising solution is to use low-dimensional random systems [10-12]. It has been shown that random multi-layers with disorder may provide directional output [10]. Directional pulsed random lasing has been also demonstrated in the photonic crystal fibre (PCF) having the hollow core filled with a suspension of $\mathrm{TiO}_{2}$ particles in a Rhodamin $6 \mathrm{G}$ dye solution [11]. In this scheme, the waveguide properties of the hollow PCF providing transverse confinement are combined with traditional bulk random material providing lasing.

*babin@iae.nsk.su; phone +7 (383) 3306939

Fiber Lasers IX: Technology, Systems, and Applications, edited by Eric C. Honea,

Proc. of SPIE Vol. 8237, 82373E - (c) 2012 SPIE · CCC code: 0277-786X/12/\$18 · doi: 10.1117/12.907965

Proc. of SPIE Vol. 8237 82373E-1 
An alternative approach of one-dimensional random laser is demonstrated recently in [12] that is based on using a conventional telecommunication fibre both for wave guiding and for random lasing exploiting the intrinsic disorder of the fibre glass structure. Indeed, the refractive index in the core of standard telecommunication fibre has submicron-scale inhomogeneities, which are randomly distributed along the fibre. Propagating light experiences Rayleigh scattering on these inhomogenities [13]. It is well known that the Rayleigh scattering (RS) sets a lower limit of losses in telecommunication fibres (amounting to $\sim 0.2 \mathrm{~dB} / \mathrm{km}$ at $\sim 1550 \mathrm{~nm}$ ). In the random laser considered here we take advantage both of light wave guiding and the Rayleigh scattering that typically is a non-desirable effect in fibre devices. Only a small fraction of the scattered radiation is reflected back into the fibre waveguide: in a single-mode telecom fibre this part amounts to $1 / 600$ of the total RS intensity that is defined by the waveguide acceptance angle. Hence, the Rayleigh backscattering coefficient is extremely small having a typical value as low as $\varepsilon=4.5 \cdot 10^{-5} \mathrm{~km}^{-1}$. This feature makes the laser system considered in [12] rather different from many conventional random lasers operating in the diffusive regime based on strong scattering. The important feature of random fibre laser [12] is that the weak randomly backscattered radiation may be amplified through the Raman effect during propagation in a long fibre waveguide thus providing feedback sufficient for lasing.

In this paper we demonstrate an important step in moving random lasers to the stage of practical applications. As it will be shown below the proposed tunable random fibre laser outperforms characteristics of the conventional Raman fibre lasers with linear cavity formed by point-action reflectors in the same fibre. Compared to other schemes of tunable Raman lasers, e.g. with ring cavity [14, 15], the developed laser appears to have much better flatness of the tuning curve in conventional telecommunication wavelength range around $1550 \mathrm{~nm}$.

\section{TUNABLE RANDOM FIBRE LASER CHARACTERISTICS}

\subsection{Experimental scheme}

Figure 1 illustrates the scheme of the proposed tunable random fibre laser based on the random distributed feedback (DFB) due to the Rayleigh scattering. The important difference to the basic scheme of the random DFB fibre laser described in [12] is that the fibre pigtailed tunable filter is introduced in the centre of the scheme. The symmetric configuration with two spans of standard single mode optical fibre (SMF-28) of total length $L=2 \times 21 \mathrm{~km}=42 \mathrm{~km}$ was used as a random laser medium (the length is $\sim 2$ times shorter than that in [12] chosen to provide maximum output power according to the calculated power distribution, see Fig.1).

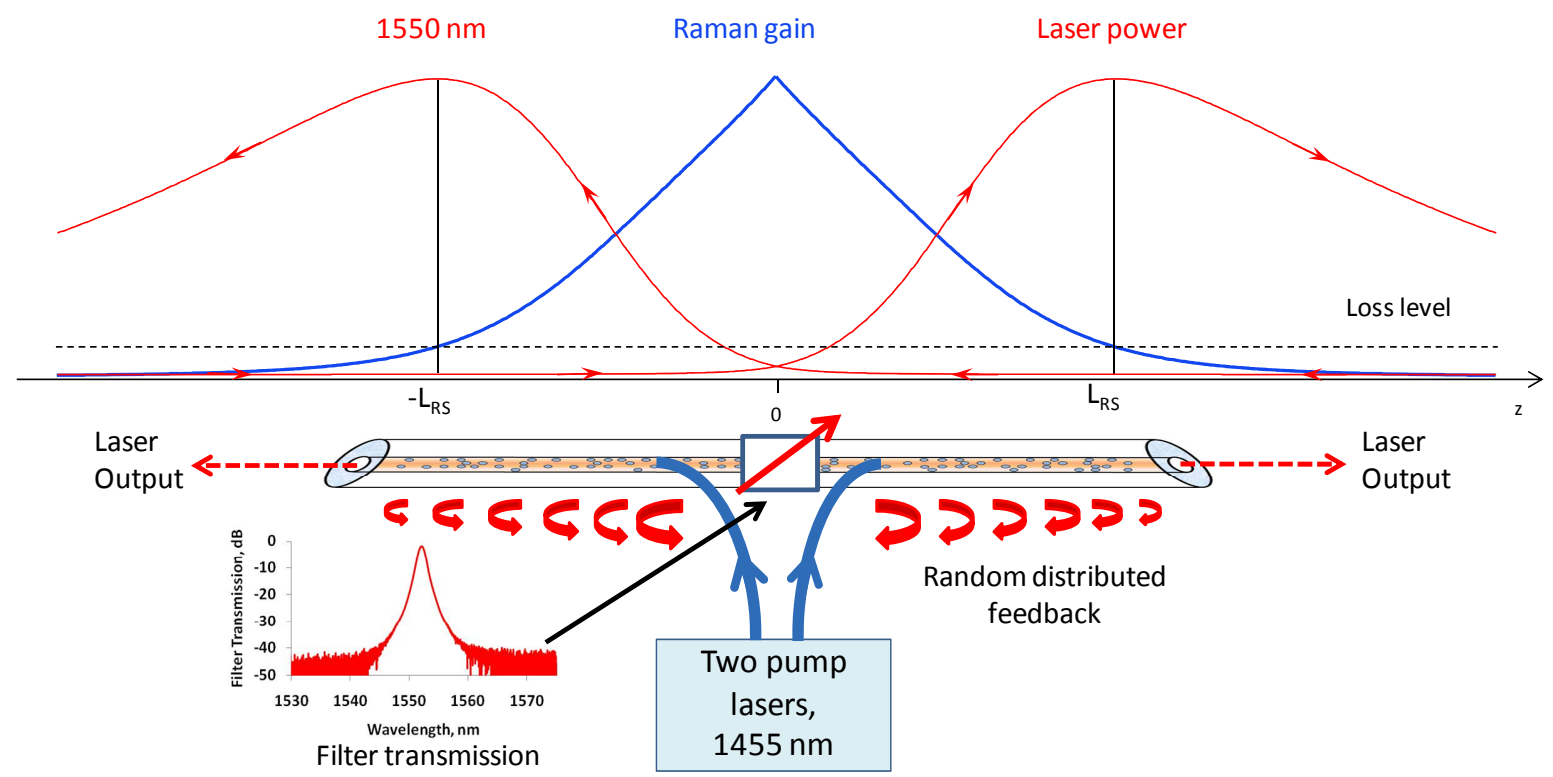

Figure 1. Schematic of the random DFB tunable fibre laser: Photons propagating in both direction are scattered, amplification is achieved by coupling two equal-power $1455 \mathrm{~nm}$ pump waves into the centre while the tunable bandpass filter in the middle set the wavelength, the fibre length is chosen to give maximum output power at fibre length just after $\mathrm{z}=\mathrm{L}_{\mathrm{RS}} \sim 20 \mathrm{~km}$. The calculated Raman gain and power distribution [12] are shown above. 
The fibre has a loss coefficient $\alpha \sim 0.045 \mathrm{~km}^{-1}(0.2 \mathrm{~dB} / \mathrm{km})$ in the transparency window of silica glasses around $1550 \mathrm{~nm}$. Two equal-power 1455-nm pumping waves are coupled at the centre through 1450/1550-nm WDM couplers in opposite directions, thus providing distributed Raman gain with coefficient $g_{R} \sim 0.39 \mathrm{~km}^{-1} \mathrm{~W}^{-1}$ at $\lambda \sim 1550 \mathrm{~nm}$. The fibre-pigtailed tunable bandpass filter inserted between the WDM couplers acts as a wavelength selective element that can be tuned over the telecom C-band (1530-1570 nm). The transmission spectrum of the filter measured by supercontinuum source is illustrated in the inset in Fig. 1 showing that the filter has $\sim 1.5 \mathrm{~nm}$ FWHM and $\sim 2 \mathrm{~dB}$ insertion loss. Angled cleaves were used at the fibre end facets to eliminate reflections and to ensure that the feedback is due to the Rayleigh scattering only. For comparison, conventional Raman laser with linear cavity in the same fibre formed by two normally cleaved fibre ends or one cleaved end and a fibre loop mirror connected to the opposite side of the cavity have been also investigated.

The laser output power and spectra are measured from both ends with a high resolution $(\sim 0.01 \mathrm{~nm})$ optical spectrum analyzer (OSA) connected via angled cleaved connector. The radio frequency (RF) spectra characterizing intensity fluctuations were also registered using photodiode and electric spectrum analyzer (ESA) with a resolution of $\sim 100 \mathrm{~Hz}$. A sampling oscilloscope with $20 \mathrm{Gsample} / \mathrm{s}$ and bandwidth of $3.5 \mathrm{GHz}$ was used to analyze the corresponding intensity behavior in time domain.

\subsection{Output characteristics}

Output power dependence measured at the left end at fixed wavelength is shown in Fig. 2 as a function of the total pump power. Note that in the symmetric system with symmetric pumping the measured output powers and spectra are equal at both fibre ends that was checked directly.

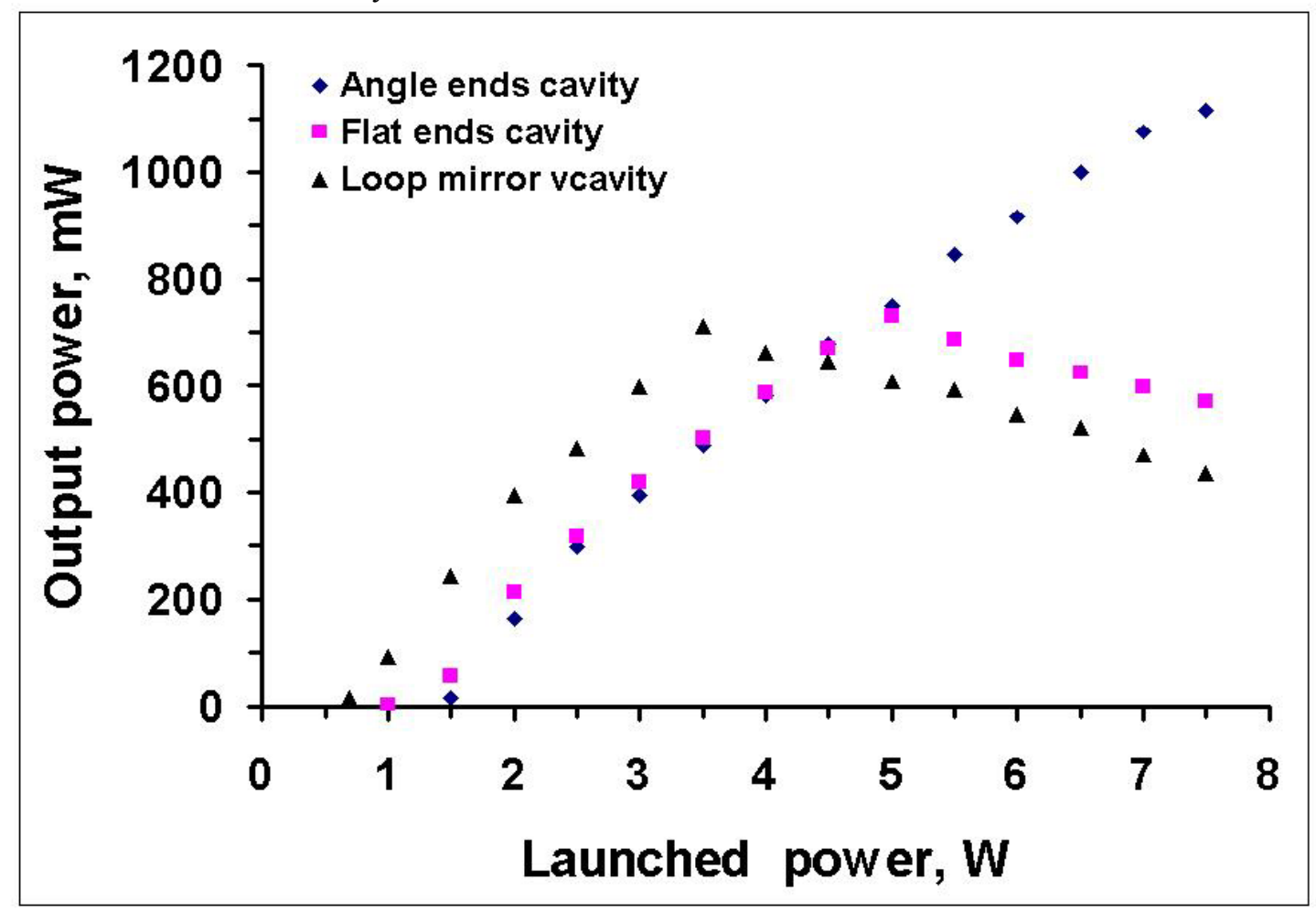

Figure 2. Random DFB fibre laser power in comparison. Left output power (at $1560 \mathrm{~nm}$ ) as a function of the total input pump power $P=2 P_{\text {in }}$ (at $1455 \mathrm{~nm}$ ). For random fibre laser (diamond points) the output power is $>1.1 \mathrm{~W}$ from

one end. For $4 \%$ cavity and loop-mirror cavity (square and triangle points respectively) the threshold is lower but maximum power is lower too. The scheme with loop mirror has only one output end so its total power is 2 times lower than in symmetric schemes.

At the threshold the generation is unstable as a result of cooperative Rayleigh-Brillouin scattering [16]. When the pump power is increased well above the threshold the laser starts to operate in the quasi-CW regime (in ms scale) with 
increasing output power nearly proportionally to the pump power. We perform a direct comparison of the RDFB laser with the conventional laser based on broadband $4 \%$ reflection from the normally cleaved fibre ends and the second laser configuration based on one cleaved fibre end and highly-reflecting fibre loop mirror connected to the other side of the same fibre. For the cavity with $4 \%$ reflection the threshold is lower and becomes much lower when the loop mirror is connected, but at high pump power the RDFB fibre laser reaches 2-times higher output power than $4 \%$ cavity amounting to $\sim 1.1 \times 2=2.2 \mathrm{~W}$ output power from two ends with $\sim 40 \%$ differential efficiency and $>4$ times higher output than that the cavity with loop mirror (that delivers laser power from one fibre end only). The observed decrease of output power in normal cavity is found to be reasoned by the second Stokes wave that starts to generate at $\sim 1650 \mathrm{~nm}$.
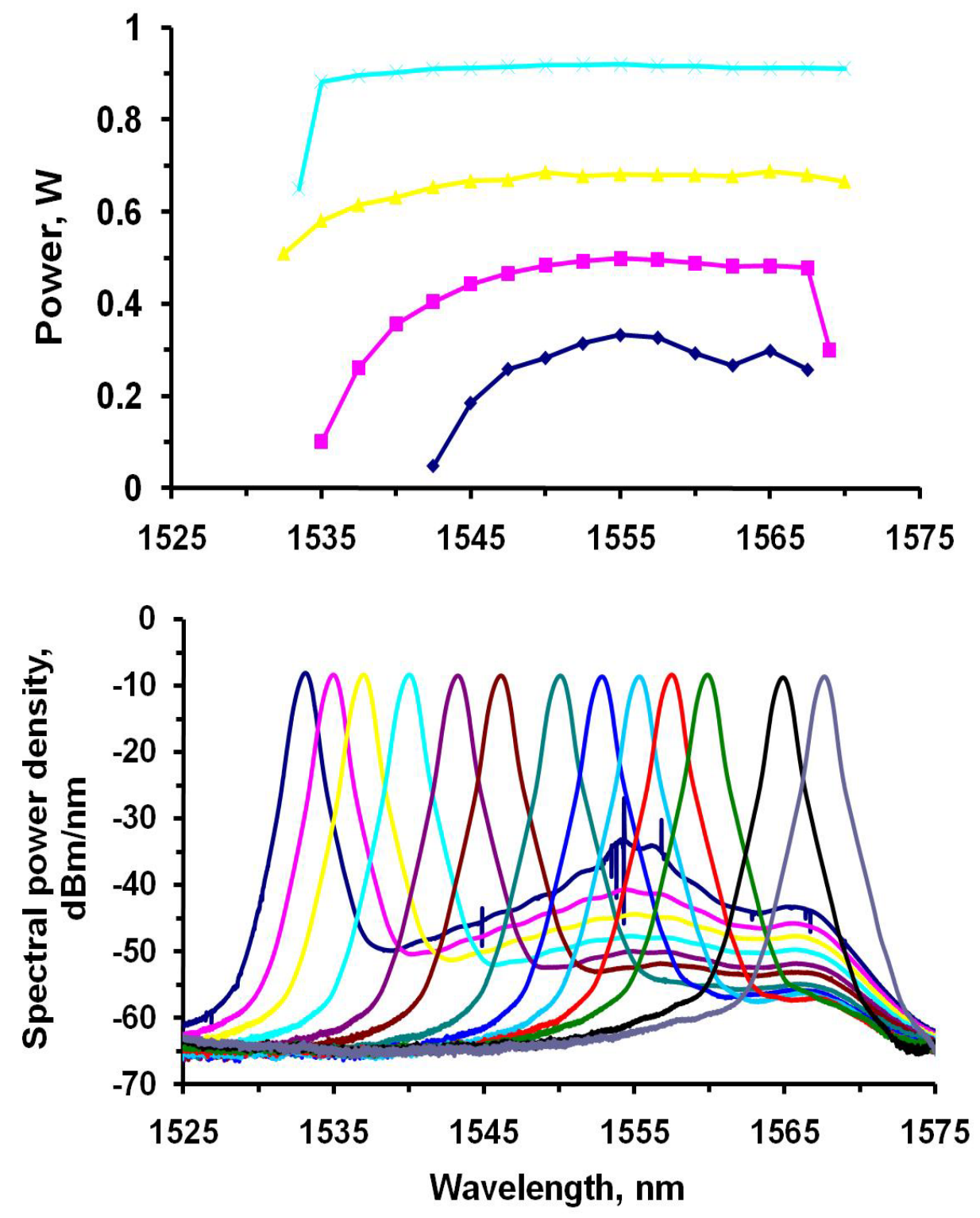

Figure 3. Random DFB fibre laser tuning range. a) Tuning curve for different total pump power in linear scale; at pump power well above the threshold the variation of the laser output power is $\sim 3 \%$ for all wavelengths in the range $1535-1570 \mathrm{~nm}$. b) Spectra at different wavelengths are shown at total pump power $6 \mathrm{~W}$. For wavelengths away from Raman gain, laser around Raman gain maxima, 1555, $1565 \mathrm{~nm}$ starts to generate. 
Figure 3a shows the output power measured at the left output end versus wavelength set by the filter. Such tuning curves were measured at different total pump powers. Figure $3 \mathrm{~b}$ shows variation of the laser output spectrum while tuning the filter at the constant total pump power of $6 \mathrm{~W}$, corresponding to the upper tuning curve of Fig.3a. In addition to the constant power value at the tuning, the generated optical spectra are $\sim 50 \mathrm{~dB}$ above the noise level for all wavelengths except the shortest one. Note that not far from the threshold, the tuning curves nearly copy the Raman gain spectrum with two maxima at 1555 and $1565 \mathrm{~nm}$ (lower curves in Fig. 3a). With increasing pump power, the tuning range broadens and the tuning curve becomes more flat. At pump power of $6 \mathrm{~W}$, the laser output power becomes constant with accuracy of $3 \%(\sim 0.1 \mathrm{~dB})$ for all wavelengths in the range 1535-1570 nm that is much better that that in conventional laser cavity in the same fibre. The reason for power decrease at wavelengths lower than $1535 \mathrm{~nm}$ is that the filter wavelength is tuned far away from the Raman gain maximum and the system starts to lase near the Raman gain maximum around $1555 \mathrm{~nm}$, just like in laser without filter [12].

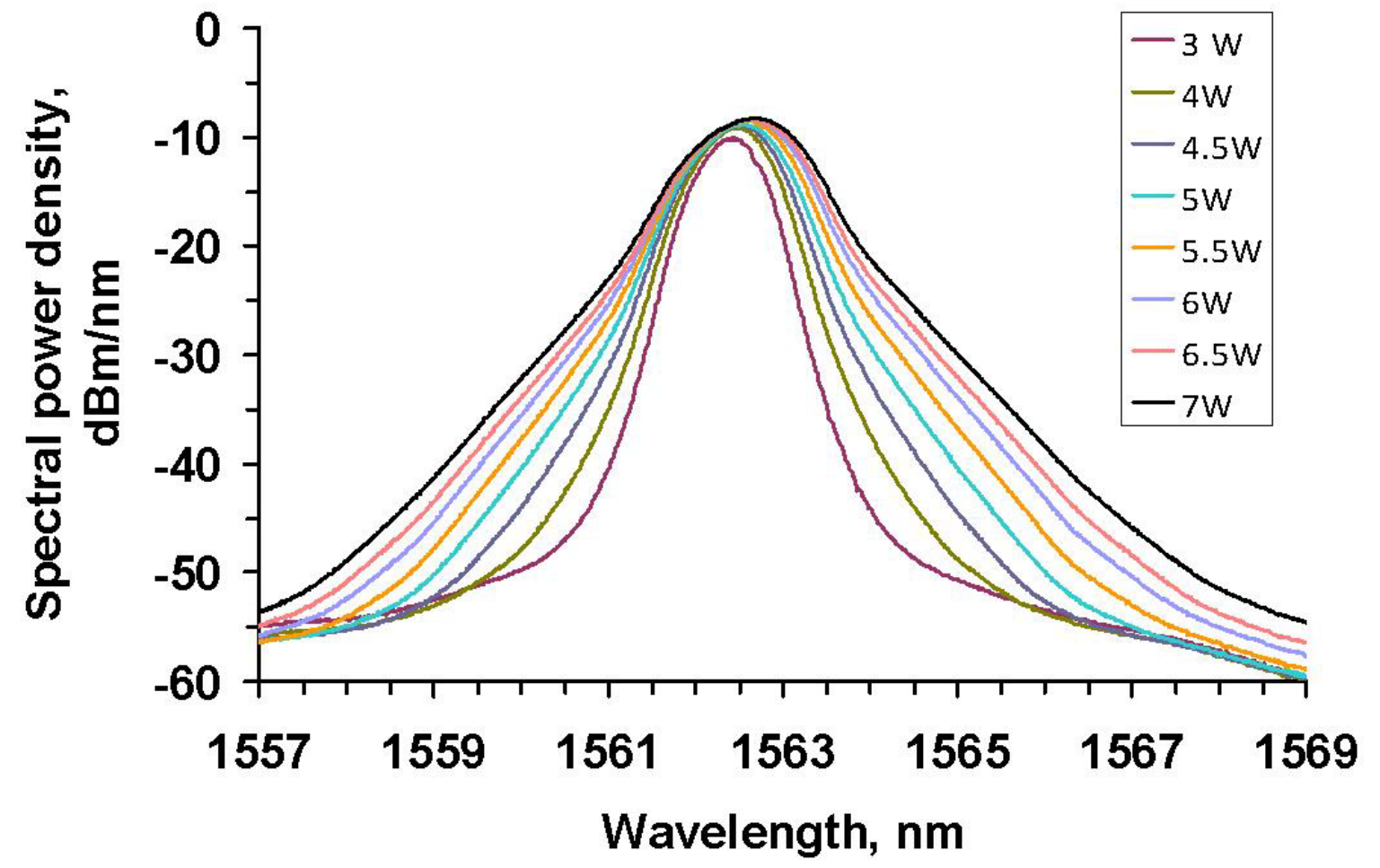

Figure 4. Random DFB fibre laser spectrum. Spectra for wavelength $1562.5 \mathrm{~nm}$ are shown at different total pump power. With increasing power, the top part of the spectrum remains nearly the same while exponential wings grow.

The measured laser output spectrum nearly copies the filter transmission near the threshold (with $1.5 \mathrm{~nm}$ FWHM), but broadens with power, see Figure 4. Note that the top part of the spectrum remains nearly the same while exponential wings grow with increasing powers, similar to the spectral broadening in conventional Raman laser defined by turbulentlike nonlinear interactions of multiple cavity modes [17].

The principal difference between the random cavity and conventional cavity is seen in the radio frequency spectrum, see Figure 5. For a conventional fibre Raman laser a clear mode structure with spacing $c / 2 L n \sim 2.4 \mathrm{kHz}$ corresponding to the round trip in the cavity with length $L=42 \mathrm{~km}$ was observed (similar to the Raman laser with highly reflecting mirrors [18]), as opposed to the random fibre laser where no mode beatings are seen. Thus, the developed tunable random fibre laser exhibits high-efficiency generation with narrow spectrum, quasi-CW output power with high-frequency intensity fluctuations having Gaussian probability density function, just like conventional fibre laser. At the same time, it has principal distinctions, namely modeless spectrum, much higher output power and outstanding flatness of the tuning curve. 


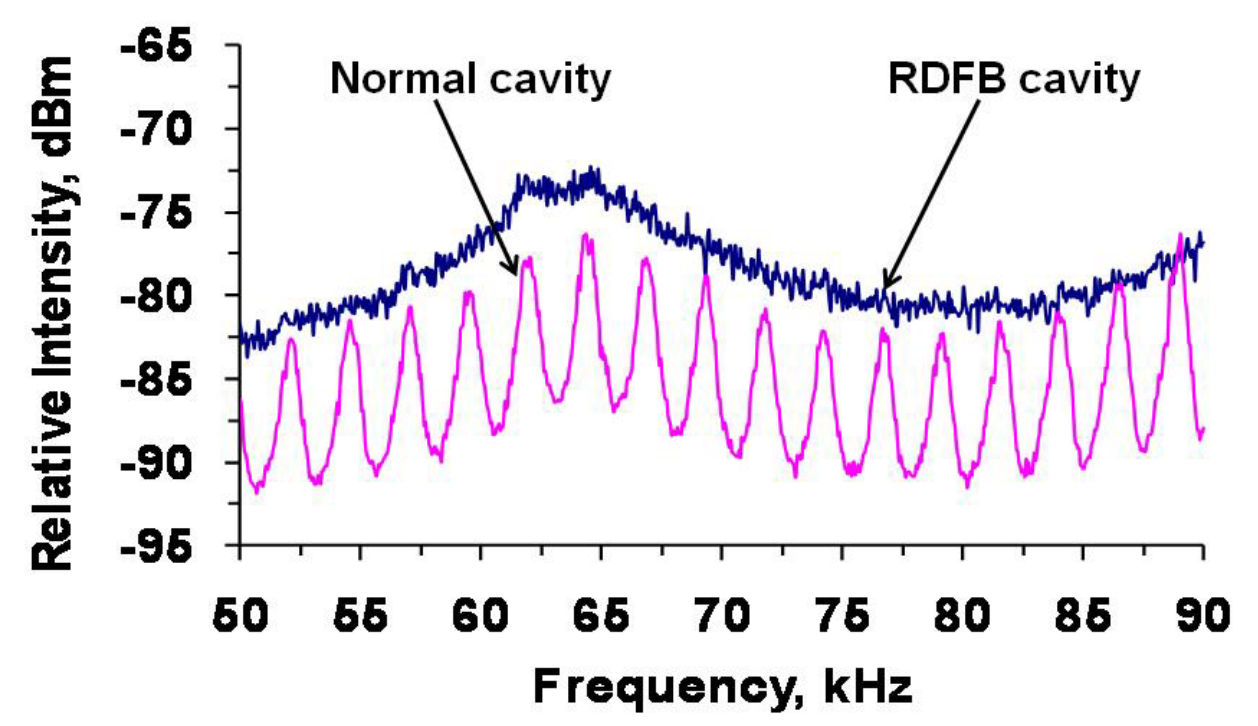

Figure 5. Random DFB fibre laser radio frequency spectrum. The spectrum is measured at left output at wavelength $\sim 1560 \mathrm{~nm}$. For random fibre laser no mode beatings are seen but for the conventional fibre Raman laser with $4 \%$ cavity a clear mode structure was observed.

\section{MODEL}

To explain the observed features we develop a simple analytical model of the random fibre laser based on RS that we only outline here. Solving the balance equations for pump power $P(z)$ and generated Stokes waves power $I_{f, b}(z)$ (fforward, b- backward) in the symmetric fibre system with two pumps of power $P_{\text {in }}$ coupled at the centre (z=0) in opposite directions like in Fig.1, one can obtain the generation efficiency in the limit of very low reflection coefficient in the fibre spans (in the first approximation replaced by point reflectors with coefficient $R<<1$ ) taking equal losses $\alpha_{p} \approx \alpha_{I}=\alpha$ :

$$
\eta=\frac{I_{\text {out }}}{P_{\text {in }}}=\exp \left[-\delta_{1}-\delta_{2}-\alpha L / 2\right] \times \frac{g_{S}}{g_{P}} \times\left\{1-\frac{1}{\sqrt{R}} \exp \left[-g_{S}(\lambda) P_{\text {in }} e^{-\delta_{1}}\left(1-e^{-\alpha L / 2}\right) / \alpha\right\}\right.
$$

where $I_{\text {out }}$ and $P_{\text {in }}$ are generated and pump power in each direction, $L / 2$ is the length of each arm, $\delta_{1}$ and $\delta_{2}$ are loss coefficients for the pump input and laser output coupling, $g_{S}$ and $g_{R}$ are the Raman gain coefficients at the pump and laser wavelength, correspondingly. The generation threshold

$$
P_{i n}^{\text {th }}(\lambda)=\frac{1}{2} \times \frac{\alpha \exp \left[-\delta_{1}\right] \ln (1 / R)}{g_{S}(\lambda)(1-\exp [-\alpha L / 2])}
$$

is wavelength sensitive due to the wavelength dependence of the Raman gain coefficient $g_{s}(\lambda)$. At the same time, the sensitivity of the threshold pump power to $R$ is logarithmically weak. Well above the threshold, for all possible generation wavelengths (satisfying condition $P_{i n}>P_{i n}^{\text {th }}(\lambda)$ ), the generation efficiency $\eta(\lambda)$ is almost independent of wavelength, since the deviation from the limiting value

$$
\eta \approx \exp \left[-\delta_{1}-\delta_{2}-\alpha L / 2\right] \times g_{S} / g_{P}
$$

becomes exponentially small at high $P_{i n}$ (second term in Eq.1). Factor $g_{S}(\lambda) / g_{P}(\lambda)$ is also almost independent of $\lambda$ and close to unity. As a result, the constant power corresponding to maximum efficiency (3) is achieved for all wavelengths being well above the threshold.

For the real random fibre laser one should take into account that the feedback is distributed, so the equations become integral and may be solved only numerically, however, the simplified physical model provides qualitative explanation 
and even rather good quantitative estimate. A comparison of the experimental random DFB laser efficiency with the proposed simple intuitive model at one calculated for RS distributed mirrors from formula (1) with $R_{\text {eff }}=\mathrm{Q}=1 / 600 \approx 0.0017$ is shown in Fig.6 demonstrating very good agreement.

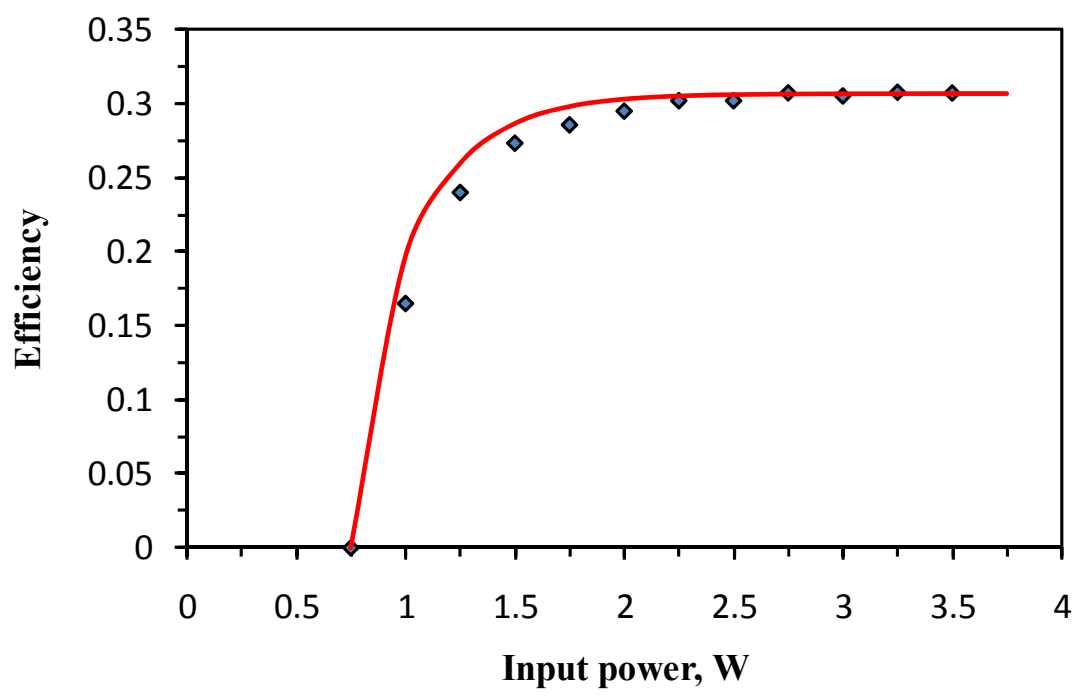

Figure 6. Random DFB fibre laser efficiency: Experiment (points) and theory (line) according to formula (1).

The main feature is exponential approach of the efficiency to the constant (maximum) value well above the threshold. The formula (1) is valid for extremely low reflection coefficient $(R<<1)$ and long $\left(L / 2 \geq \alpha^{-1}\right)$ fibre pumped by highpower radiation $\left(g_{R} P_{i n} / \alpha>>\ln [1 / R] / 2\right)$. The maximum possible conversion efficiency is $\eta_{\max } \sim \exp (-\alpha L / 2)$ estimated as 0.4 in our case $(L / 2=21 \mathrm{~km})$, and we are close to this value in the experiment.

\section{DISCUSSION}

The demonstrated tunable random DFB fibre laser exhibits outstanding characteristics, namely:

(i) much higher output power of the generated Stokes wave than the corresponding conventional Raman fibre laser with cavity formed by $4 \%$ Fresnel reflection or loop mirror in the same fibre;

(ii) very flat tuning curve covering $>35 \mathrm{~nm}$ despite rather non-uniform Raman gain spectral profile having $>10$ $\mathrm{dB}$ variation of the gain coefficient.

At $1455 \mathrm{~nm}$ pumping the Raman gain profile has two maxima at 1555 and $1655 \mathrm{~nm}$ which are clearly seen in the spectrum of amplified spontaneous scattering (corresponding to background in Fig.3b). Note that the long-wavelength limit $(1570 \mathrm{~nm})$ is defined by the operating range of the filter and may be further extended. The achieved flatness of $3 \%$ is much better than that one in conventional laser cavity tested with the same fibre, as well as in other tunable Raman fibre laser configurations operating in telecom spectral band $[14,15]$.

The highest achievable output power for the generated Stokes wave is defined by the threshold for the second Stokes wave generation that is much higher in the scheme with RS-based random distributed feedback compared to the schemes with point-action reflectors in the same fibre. Moreover, the random DFB fibre laser has higher slope efficiency in spite of sufficiently higher threshold (see Fig.2), therefore its output power exceeds the power of the conventional Raman laser with $4 \%$ cavity even before reaching the second Stokes threshold. This fundamental experimental observation is supported by the formula (1): in the limit of extremely low reflection coefficient (estimated as $R_{\text {eff }} 0.0017$ for RSfeedback) and long $\left(l \geq \alpha^{-l}\right)$ fibre pumped by high-power radiation $\left(g R P_{i n} / \alpha>>\ln (1 / R) / 2 \sim 3\right)$ all conditions satisfied in our experiment, the second term in square brackets is vanishingly small and the efficiency tends to its limiting value (3) that does not depend on $\lambda$ and corresponds to the maximum possible conversion efficiency. It is defined by factor $\eta_{\max } \sim \exp (-\alpha L / 2)$ estimated as 0.4 in our case $(L / 2=21 \mathrm{~km})$ neglecting insertion losses and difference in Raman gain coefficients. In the experiment we are close to this value (see Fig.6). In addition to extremely low reflection coefficient the developed random fibre laser is characterized by specific laser power distribution along the cavity with very low power in the center where filter is placed (see numerically calculated axial distribution of the laser power taking into 
consideration distributed nature of the RS-based feedback [12], Fig. 1). As a result, the nonlinear loss due to filtering being sensitive to the broadening of the spectrum is lower for the proposed random laser because of much lower local power in the middle point where the filter is placed. Another important feature of the random DFB laser is selfadjustment of the effective distributed cavity at high conversion efficiency: high output power depletes the pump wave thus shortening the length $2 L_{R S}$ of the effective cavity where back reflected light is amplified. This effect makes it possible to use even shorter fibres providing higher efficiency in correspondence with lower linear loss factor, $\eta_{\max } \sim \exp (-\alpha L / 2)$.

Note that the proposed mechanism of wavelength independent flat power generation in pure random lasers can be applied to development of multi-wavelength lasers. In particular, our model explains the observed flat power distribution recently observed in the multi-wavelength Raman fibre laser [19] with a cavity formed by an array of 22 narrowband fibre Bragg gratings (i.e. highly reflective mirrors operating at different wavelengths) from one side and the RS-based random distributed mirror from the other side of the fibre. Competition of random spectral components which utilize the same pump should lead to their self-organization resulting in equalized power distribution. These new effects are important for description of spectral and noise characteristics which is a challenging goal for the development of random fibre laser theory.

\section{CONCLUSION}

Thus we have demonstrated tunable control of the properties of random lasers with rather simple engineering design solution albeit rich and complex underlying physical mechanisms. The proposed tunable random fibre laser demonstrates outstanding performance compared both to other types of random lasers and to same category conventional fibre lasers with point-action reflectors. It is shown that similar principle may be applied for development of multi-wavelength lasers. The demonstration of a broad-range tunable random fibre laser with good spatial and spectral performance represents a significant milestone for random laser science and adds a new dimension to the applications of disorderbased light sources. The demonstrated laser presents a new object for fundamental research as well as a practical device with high performance and simple design implemented in standard optical fibre offering new applications in optical communication, sensing and secure communications.

\section{ACKNOLEDGMENTS}

Authors acknowledge the financial support of the Ministry of Education and Science and integration program of the Siberian Branch of the Russian Academy of Sciences, Leverhulme Trust, HEFCE, The Royal Society, and FP-7-IRSES program.

\section{REFERENCES}

[1] Wiersma D. S., "The smallest random laser", Nature 406, 132-135 (2000).

[2] Wiersma D. S., "The physics and applications of random lasers", Nature Physics 4, 359-367 (2008).

[3] Cao H., "Random lasers with coherent feedback," in [Topics in Applied Physics, vol. 82: Optical Properties of Nanostructured Random Media, V. M. Shalaev, ed.], Springer-Verlag, Berlin (2002).

[4] Cao H., "Review on latest developments in random lasers with coherent feedback", J. Phys. A: Math. Gen. 38, 10497-10535 (2005).

[5] Noginov M. A.,[Solid-State Random Lasers], Springer, Berlin (2005).

[6] Fallert J. et al., "Co-existence of strongly and weakly localized random laser modes", Nature Photonics 3, 279$282(2009)$.

[7] Wiersma D. S., "Laser physics: Random lasers explained?", Nature Photonics 3, 246-248 (2009).

[8] Letokhov V.S., "Generation of light by a scattering medium with negative resonance absorption", Sov. Phys. JETP 26, 835-840 (1968).

[9] Markushev V. M., Zolin V. F. \& Briskina Ch. M., "Powder laser", Zh. Prikl. Spektrosk. 45, 847-850 (1986).

[10] Milner V., Genack A. Z., "Photon localization laser: Low-threshold lasing in a random amplifying layered medium via wave localization", Phys. Rev. Lett. 94, 073901 (2005). 
[11] de Matos C.J.S., Menezes L.S., Brito-Silva A.M., Martinez Gamez M.A., Gomes A.S.L., Araujo C.B., "Random Fiber Laser", Phys. Rev. Lett. 99, 153903 (2007).

[12] Turitsyn S.K., Babin S.A., El-Taher A.E., Harper P., Churkin D.V., Kablukov S.I., Ania-Castañón J.D., Karalekas V., Podivilov E.V., "Random Distributed Feedback Fibre Laser", Nature Photonics 4, 231-235 (2010).

[13] Lord Rayleigh (Strutt, J. W.), "On the transmission of light through an atmosphere containing small particles in suspension and on the origin of the blue sky", Philos. Mag. 47, 375-384 (1899).

[14] Reeves-Hall P. C., Taylor J. R., "Wavelength tunable CW Raman fibre ring laser operating at 1486-1551 nm", Electron. Lett. 37, 491-492 (2001).

[15] Yeo K. S., Mahdi M. A., Mohamad H., Hitam S., Mokhtar M., "Widely Tunable Raman ring laser using highly nonlinear fibre", Laser Physics 19, 2200-2203 (2009).

[16] Ravet G., Fotiadi A.A., Blondel M. and Megret P., "Passive Q-switching in all-fibre Raman laser with distributed Rayleigh feedback", Electr. Lett. 40, 528-529 (2004).

[17]Babin S.A., Karalekas V., Harper P., Podivilov E.V., Mezentsev V.K., Ania-Castañón J.D., Turitsyn S.K., "Turbulent broadening of optical spectra in ultralong Raman fibre lasers", Phys. Rev. A. 77(3), 033803 (2008).

[18] Babin S.A., Karalekas V., Harper P., Podivilov E.V., Mezentsev V.K., Ania-Castañón J.D., Turitsyn S.K., "Experimental demonstration of mode structure in ultra-long Raman fibre lasers", Optics Letters 32(9), 11351137 (2007).

[19]El-Taher A. E., Harper P., Babin S. A., Churkin D. V., Podivilov E. V., Ania-Castanon J. D., Turitsyn S. K. , "Effect of Rayleigh-scattering distributed feedback on multiwavelength Raman fibre laser generation", Optics Letters 36(2), 130-132 (2011). 